\title{
The Amino Acid Use in Cultures of Phase I Bordetella pertussis During Growth in Chemically Defined Media
}

\author{
By ANN H. VAJDIC, M. GOLDNER AND R. J. WILSON \\ Connaught Medical Research Laboratories, \\ University of Toronto, Toronto, Canada
}

(Received 28 February 1966)

\begin{abstract}
SUMMARY
The primary metabolic importance of glutamic acid for phase I Bordetella pertussis was illustrated by its rapid use during the exponential stage of the growth in a chemically defined medium. The pattern of uptake of proline, in particular, and of glutamine resembled that of glutamic acid; this was explained in terms of the conversion of proline and glutamine to glutamic acid. Other substantially used amino acids (alanine, glycine, histidine, serine) were taken up when the readily available sources of glutamic acid became exhausted and the culture entered the stationary phase of growth. The results given show the order of uptake for the amino acids in relation to the growth stage of the culture. No relation was apparent between the amino acid uptake and the immunogenicity of the organisms throughout the growth period.
\end{abstract}

\section{INTRODUCTION}

The previous paper (Goldner, Jakus, Rhodes \& Wilson, 1966) was concerned with the amino acid composition of batch culture media after the growth of phase I Bordetella pertussis in the chemically defined medium (Wilson, 1963) had reached the stationary phase. A determination of amino acid requirements through the growth period of the batch culture would provide a more dynamic picture of amino acid utilization in relation to the growth stage. In addition, the determination of the antigenicity of the organisms at various stages of growth might reveal a relation between the uptake of a particular amino acid and a change in the immunogenicity of the organisms.

\section{METHODS}

Media and methods were as previously described (Wilson, 1963; Goldner et al. 1966), with the following exceptions in the chromatographic technique. The staining procedure for the chromatograms differed in that these were dipped in an acetone solution of ninhydrin. The ninhydrin reagent was prepared after the method of Block (1950) except for the use of acetone instead of isopropanol as diluting solvent for the ninhydrin stock solution (R. Hilsheimer, personal communication). Specific staining for cystine and proline was done according to the following methods: the sodium nitroprusside + sodium cyanide (Toennies \& Kolb, 1951) reagent was used for cystine, and the isatin in acetone reagent recommended by Block, Durrum \& Zweig (1958) for proline.

Antigenic studies. Vaccines were prepared from cultures at different stages of growth. Samples of replicate culture tubes were pooled, adjusted to contain $5 \times 10^{9}$ 
organisms $/ \mathrm{ml}$., inactivated with $\mathbf{0 . 0 7 \%}$ formalin and tested for immunogenicity (Worcester \& Wilson, 1943) by the mouse protection test. Bacterial concentrations were adjusted by addition or removal of culture supernatant fluid (Pekárek \& Stejskal, 1962).

\section{RESULTS}

In the results given below, the terms 'use' or 'utilization' relate to the disappearance or decrease in intensity of spots on the chromatograms.

Nine samples for paper chromatography were taken during the lag, exponential and stationary phases up to $\mathbf{7 2} \mathrm{hr}$ (Fig. 1). of growth in defined amino acid medium A (Goldner et al. 1966). Determinations of the use of the various amino acids in relation to the growth stages are presented in Fig. 2.

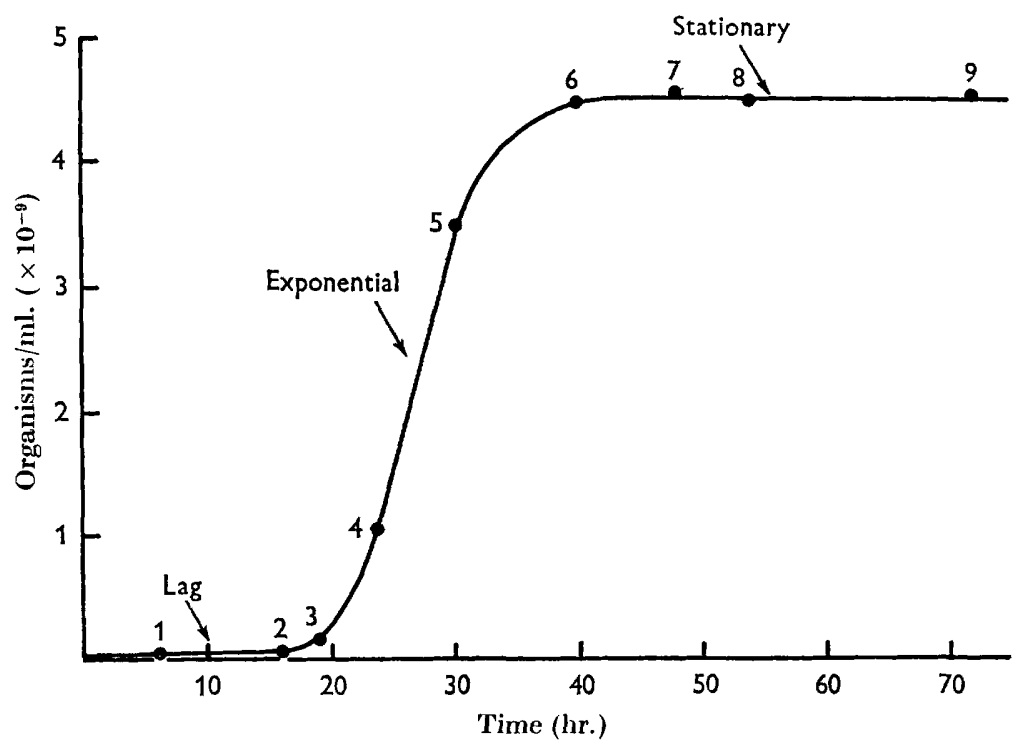

Fig. 1. Growth curve of Bordetella pertussis in chemically defined amino acid medium sample no. at time $(\mathrm{hr}): 1,6 ; 2,16 ; 3,19 ; 4,24 ; 5,30 ; 6,40 ; 7,48 ; 8,54 ; 9,72$.

Glutathione was moderately used during the exponential period, and this increased after the beginning of the stationary phase. Cystine + aspartic acid began to be decreased at about the start of the stationary phase. No cystine was detected with the specific stain after $48 \mathrm{hr}$. Aspartic acid was used only to a moderate extent. Glutamic acid was moderately used by the beginning of the exponential phase and was depleted before the beginning of the stationary phase. Proline showed a comparable pattern except that the decrease was more rapid. Histidine +lysine was moderately used and then only at the start of the stationary phase; previous results (Goldner et al. 1966) had shown that this depended on the use of the histidine. The hydroxyproline + threonine and tyrosine + tryptophan spots decreased to the same extent; unlike histidine, these components cannot be considered important. The glutamine + arginine spot showed a progressive decrease in intensity from the beginning of the exponential phase. This result was due almost entirely to disappearance 
of glutamine (Goldner et al. 1966). Decrease in serine + glycine and alanine began at the end of the exponential phase and continued throughout the stationary phase. The remaining amino acids in the chemically defined medium (methionine + valine,

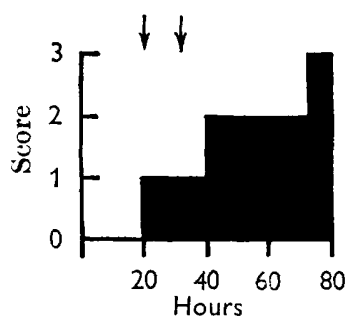

Glutathione

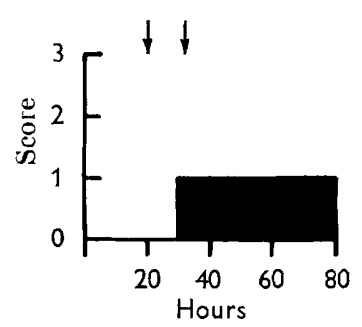

Histidine +lysine

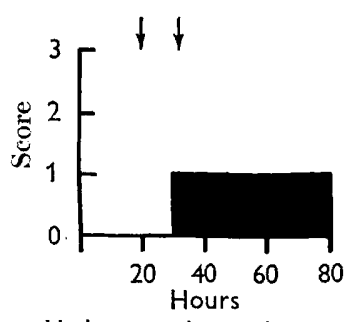

Hydroxyproline+threonine Tyrosine+tryptophan

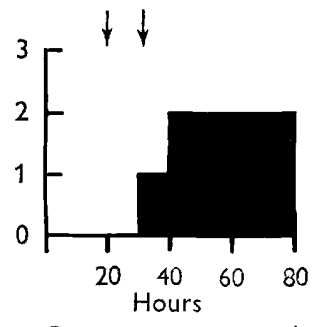

Cystine +aspartic acid

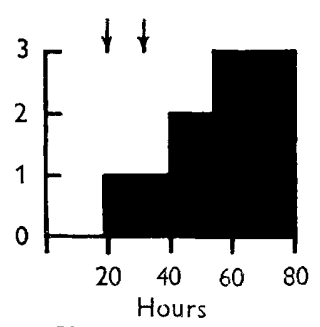

Glutamine-arginine

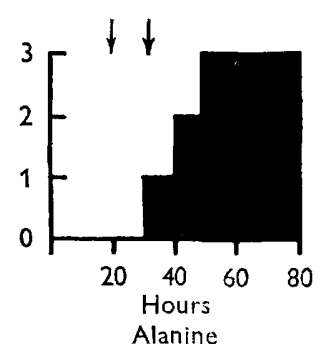

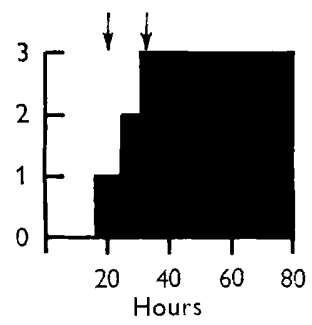

Glutamic acid

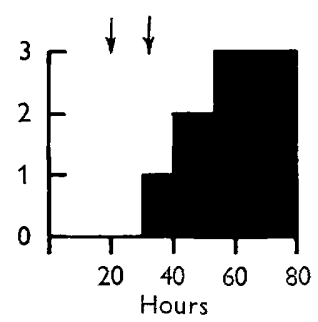

Serine+glycine

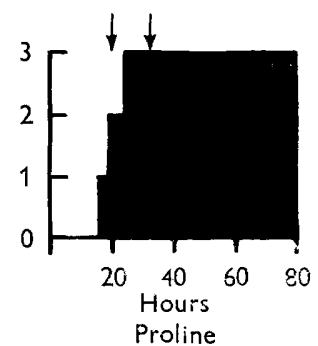

Fig. 2. Amino acid use by phase I Bordetella pertussis during growth in the chemically defined amino acid medium $A$. The individual and mixed components are grouped to correspond as closely as possible with their sequence in the chromatographic separation. Percentage utilization expressed in the form of scores, $0-15 \%=0 ; 16-45 \%=1$; $46-75 \%=2 ; 76-100 \%=3$. Score of $0=$ methionine + valine, phenylalanine, isoleucine + leucine. *Time between the arrows represents the exponential phase of growth.

Table 1. Disappearance (usage) of amino acids from defined amino acid culture medium A during batch culture of Phase I Bordetella pertussis

Amino acid

Glutamic acid, proline

Alanine, cystine,

Glycine, serine

Histidine

Aspartic acid, threonine, tryptophan, tyrosine

Organic sulphur source Glutathione

Cystine
Growth stage

Exponential phase

Stationary phase (decelerating phase)

Stationary phase (decelerating phase)

Stationary phase

Exponential phase

Stationary phase (decelerating phase) 
phenylalanine, isoleucine + leucine) were only slightly used, if at all. The preferential order of amino acid disappearance is given in Table 1.

The antigenicity of organisms harvested at the stationary phase $(48 \mathrm{hr})$ was compared to those harvested at the early $(24 \mathrm{hr})$, middle $(27 \mathrm{hr})$ and late $(30 \mathrm{hr})$ parts of the exponential phases, and the deceleration $(\mathbf{3 5} \mathrm{hr})$ phase. The immunogenicity of the vaccines at the different stages, based on the results of a duplicate set of mouse protection tests against the U.S. Standard Pertussis Vaccine, agreed within a twofold range with that observed with the vaccines prepared from organisms at the stage of the stationary phase.

\section{DISCUSSION}

Previous reports about Bordetella pertussis and related organisms (Jebb \& Tomlinson, 1951; Fukumi, Sayama, Tomizawa \& Uchida, 1953) showed glutamic acid to be of prime importance as a source of carbon, nitrogen and energy. In the present work glutamic acid was one of the first amino acids to disappear in cultures of phase I $\boldsymbol{B}$. pertussis organisms. Proline was also one of the earliest to be used showing a pattern of disappearance from the medium similar to that for glutamic acid. In view of this result, and the findings of Fukumi et al. (1953) that proline can replace glutamic acid for growth, proline may be converted to glutamic acid at an early growth stage. Glutamine may also be progressively converted to glutamic acid, particularly after the original supplies of glutamic acid and proline have been exhausted. If serine + glycine and alanine are converted to, or substituted for, glutamic acid in the defined media, they may be expected to become of value only after extensive use of glutamic acid, proline and glutamine. This appears to be the case since they were not used to a major extent until the end of the exponential stage. Thus, the culture entered the stationary phase during the period when most of the disappearance of these components occurred. The amount of histidine in the medium also decreased at a time coinciding with the complete utilization of glutamic acid.

Jebb \& Tomlinson (1951) observed that washed suspensions of Bordetella pertussis oxidized aspartic acid at a lower rate than glutamic acid, and that oxaloacetic acid was not oxidized. The present observations are in accord with this; aspartic acid was used only in the stationary phase and to a moderate extent. If only L-aspartic acid were used, the rapidity and even the extent of its use would still not be as much as that of glutamic acid. Glutathione appeared to provide the primary source of organic sulphur; its use corresponded with the start of the exponential period.

The results for the remaining amino acid components of the defined medium $A$ confirm those recorded in Goldner et al. (1966), which indicated little or no usage by the end of the growth period.

No relation between antigenicity and amino acid uptake was apparent. Pekárek \& Stejskal (1962) observed that the protective properties of their vaccines, grown in a casein hydrolysate medium, were highest in the exponential phase. At this phase of growth, under our conditions, the uptake of glutamic acid and proline predominated and higher amounts of glutamic acid and proline in the medium resulted in increased antigenicity in cultures harvested in the stationary phase (Goldner et al. 1966). 
The authors wish to thank Dr J. M. Corkill for performing the mouse protection tests, and Drs H. Pivnick and A. H. W. Hauschild for their advice on the manuscript.

\section{REFERENCES}

Block, R. J. (1950). Estimation of amino acids and amines on paper chromatograms. Anal. Chem. 22, 1327.

Block, R. J., Durrum, E. L. \& Zweig, G. (1958). Amino acids, amines and proteins. In A Manual of Paper Chromatography and Paper Electrophoresis, p. 110. New York: Academic Press Inc.

Fukumi, H., Sayama, E., Tomizawa, J. \& Uchida, T. (1953). Nutritional requiremen s and respiratory pattern of pertussis-parapertussis-bronchisepticus group of microorganisms. Jap. J. med. Sci. Biol. 6, 587.

Goldner, M., Jakus, C. M., Rhodes, H. K. \& Wilson, R. J. (1966). The amino acid utilization by phase I Bordetella pertussis in a chemically defined medium. J. gen. Microbiol. 44, 439.

Jевв, W. H. H. \& Tomurnson, A. H. (1951). The catabolic activity of washed suspensions of Haemophilus pertussis. J. gen. Microbiol. 5, 951.

Pekárek, J. \& Stejskal, A. (1962). Preparation of non-reactive and non-sensitising vaccine. Round Table Conference on Pertussis Immunisation, Prague. Vol. 1, p. 1.

Toennies, G. \& Kolb, J. J. (1951). Techniques and reagents for paper chromatography. Anal. Chem. 23, 823.

WrLson, R. J. (1963). Cultivation of Bordetella pertussis (phase I) in a chemically-defined medium. Can. J. pub. Hlth, 54, 518.

Worcester, J. \& Wilson, E. B. (1943). A table determining LD 50 or the fifty percent end-point. Proc. natn. Acad. Sci., U.S.A. 29, 207. 